\title{
Helicobacter Pylori and its Determinations on Gastric Biopsies
}

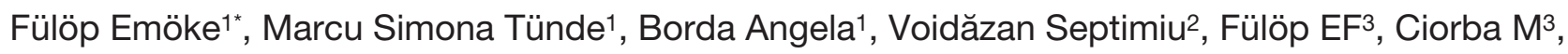 \\ Mocan Simona ${ }^{4}$
}

\author{
1 Department of Histology, University of Medicine and Pharmacy of Tîrgu Mureș, Romania \\ 2 Department of Epidemiology, University of Medicine and Pharmacy of Tîrgu Mureș, Romania \\ ${ }^{3}$ Department of Internal Medicine, University of Medicine and Pharmacy of Tîrgu Mureș, Romania \\ 4 Department of Pathology, Emergency County Hospital of Tîrgu Mureș, Romania
}

\begin{abstract}
Background and Aims. Gastric cancer, because of its aggressive evolution and the high mortality associated with it, remains one of the most debated subjects in medical literature with Helicobacter pylori (HP) as a major risk factor. Chronic inflammation caused by HP infection represents the initial site of the predisposing and afterwards premalignant lesions for gastric carcinoma. The purpose of this study was to evaluate the prevalence of HP infection, of predisposing and premalignant lesions on gastric biopsies, as well as to identify the correlations between them. Material and method. A retrospective cross-sectional study was performed on gastric biopsies collected endoscopically from a single region, antrum or corpus, and from different regions, between January 2012 and July 2014. Incidence of HP infection, of predisposing and premalignant gastric lesions, the correlation of HP infection and these lesions, were evaluated. Results. HP infection was diagnosed in $32.81 \%$. Predisposing and premalignant lesions were present in $53.64 \%$ of biopsies with most of them in the antrum. HP infection stands out for the under 50 yo group $(\mathrm{p}=0.001)$. No correlation between frequency of HP infection and predisposing and premalignant lesions was observed. Conclusions. Prevalence of HP infection in our study suggests that besides HP infection, other factors are also involved in gastric cancer development. Biopsies from different regions of the gastric mucosa do not offer extra information regarding HP infection prevalence but may be helpful in evaluating incidence and extension of predisposing and premalignant lesions.
\end{abstract}

Keywords: Helicobacter pylori infection, epidemiology, gastric cancer, risk factors, precursor lesions

Received: 14 August 2015 / Accepted: 03 September 2015

\section{Introduction}

Gastric cancer remains one of the most debated subjects in medical literature, firstly because of its aggressive evolution and because of the high mortality associated with it, despite all international efforts for improving primary and secondary prevention [1-3]. The pathogenesis of intestinal type gastric cancer involves different risk factors that lead to predisposing lesions (multifocal atrophy, intestinal metaplasia, pseudopyloric metaplasia) and premalignant lesions (displasia or intraepithelial metaplasia). Among the risk factors involved in the development of gastric carcinoma, besides environmental factors (alcohol use, salty foods, smoking and chronic biliary reflux) and genetic factors, Helicobacter pylori (HP) is considered a major risk factor, declared by $\mathrm{WHO}$ in the $1^{\text {st }}$ class of oncogenic risk and reconfirmed by the International Agency for Research on Cancer (IARC) [4-6]. The pathogen acts in two directions on epithelial cells: an indirect one, through inflammation and a direct one, by inducing genetic mutations [7]. In the first stage, the chronic inflammation caused by HP infection only affects the gastric antrum. Once the disease evolves, the chronic inflammation extends to the corpus and this will represent the initial site of the predisposing and afterwards premalignant lesions for gastric carcinoma [8-10].

* Correspondence to: Emőke Fülöp

E-mail: emifulop@yahoo.com
The purpose of this study was evaluating the prevalence of HP infection, of the predisposing and premalignant lesions, as diagnosed on gastric biopsies, identifying correlations between the infection and the lesions it determines in the gastric mucosa.

\section{Material and method}

A retrospective cross-sectional study was performed. The study material was selected from from all endoscopically prelevated gastric biopsies for diagnosis purposes and processed in the Pathology Department of the Tîrgu Mureş County Emergency Clinical Hospital, between January 2012 and July 2014. The study material included both biopsies obtained from a single gastric region, antrum or corporeal region, and those taken from different regions, but at least one from the antrum and corpus, processed and labeled accordingly. Cases submitted with material improper for diagnosis, insufficient material, and those diagnosed with benign and malignant tumors were all excluded.

Evaluation of HP infection was done in both HE (hematoxylin and eosin) stained slides and Giemsa stained slides, taking in consideration its presence or absence.

The predisposing lesions were evaluated in HE (hematoxylin and eosin) and PAS-AB (Periodic acid-Schiff-Alcian Blue) stained slides. The phenotype of the followed lesions was glandular atrophy (A), intestinal metaplasia (IM) and pseudopyloric metaplasia (PPM). In the category of glandular atrophy we included both nonmetaplastic atro- 
phy (the replacement of normal glands particular to each gastric region with fibrous tissue from the lamina propria) and metaplastic atrophy (replacement of the native glands with metaplastic ones). In the category of intestinal metaplasia we included both phenotypes, complete intestinal metaplasia - small bowel type and incomplete intestinal metaplasia - large bowel type. Pseudopyloric metaplasia was evaluated only on those biopsies obtained from the corporeal region, represented by antral glands within the oxyntic mucosa.

For premalignant lesions, intraepithelial neoplasia (IEN), we determined the following categories: negative for intraepithelial neoplasia (N-IEN), indefinite for intraepithelial neoplasia (I-IEN- foveolar hyperproliferation and hyperproliferative intestinal metaplasia), low grade intraepithelial neoplasia (LG-IEN), high grade intraepithelial neoplasia (HG-IEN).

Each parameter was evaluated by taking in account the gastric region from which the biopsy was taken. If the biopsies were taken from two different regions, evaluation was done separately considering the provenance of the samples.

In completing the evaluation of the relationship between HP infection and the lesions induced by it, we also took in account the gender and age of the patients. Because the study included only adults, the age groups covered 10 years each, the first one being between 20-29 yo while the last reffered to those patients $>90$ yo. We also conducted a comparative study between the frequency of HP infection, predisposing and premalignant lesions for patients under the age 50 and those over this respective age.

Statistical analysis was performed using the MedCalc Software, Version 12.5.0.0. Data were considered as nominal or quantitative variables. Nominal variables were characterized using frequencies. A chi-square test was used for comparison of nominal variable frequencies. The level of statistical significance was set at $\mathrm{p}<0.05$.

The study was approved by the Ethics Committee of Emergency County Hospital Tîrgu Mures under the registration number 6842 / 01.04.2015.

\section{Results}

The study included 2530 biopsies, of which 1793 (70.87\%) were taken exclusively from the antrum, 97 (3.83\%) from the corpus and $640(25.3 \%)$ from two different gastric regions, one antral and one corporeal.

HP infection was found in 830 (32.81\%) of these cases, the frequency for each region being stated in the table below (Table I) (Figure 1).

No statistically significant difference was found concerning gender distribution of HP patients $(\mathrm{p}=0.7)$, the female to male ratio was $1.5: 1$. The mean age of HP positive patients was 51.85 years old. Following statistical evaluation, we found a statistically significant correlation between HP infection and patient age $(\mathrm{p}=0.007)$, the infection being more frequent in the 50-59 yo (14.1\%), 60-69 yo $(26.39 \%)$ and $70-79$ yo $(21.9 \%)$ age groups. The results of the study show that HP infection was found in $23 \%$ of patients under the age of 50 and in $77 \%$ of patients older than 50 years.

Predisposing and premalignant lesions were diagnosed in 1357 (53.64\%) of biopsies. 792 (58.36\%) of cases were diagnosed based on biopsies taken from a single mucosal region while 565 (41.64\%) of cases were diagnosed on biopsies from two different regions. The result of the study regarding the location of the lesions in the different areas of the gastric mucosa shows that in 1078 (79.26\%) of cases the lesions were found only in the antrum, in 189 (13.9\%)

Table I. The incidence of HP infection in biopsies obtained from a single gastric region (I) and those from two different regions (II) of the gastric mucosa.

\begin{tabular}{lcc}
\hline p-0.35 & \multicolumn{2}{c}{$\mathrm{HP+}$} \\
& $\mathrm{I}$ & $\mathrm{I}$ \\
\hline antrum & 564 & 28 \\
& $(29.71 \%)$ & $(3.63 \%)$ \\
antrum and corpus & - & 218 \\
& & $(30.64 \%)$ \\
corpus & 14 & 6 \\
Total & $(0.74 \%)$ & $(0.9 \%)$ \\
& $578(30.45 \%)$ & 252 \\
& & $(35.17)$ \\
\hline
\end{tabular}

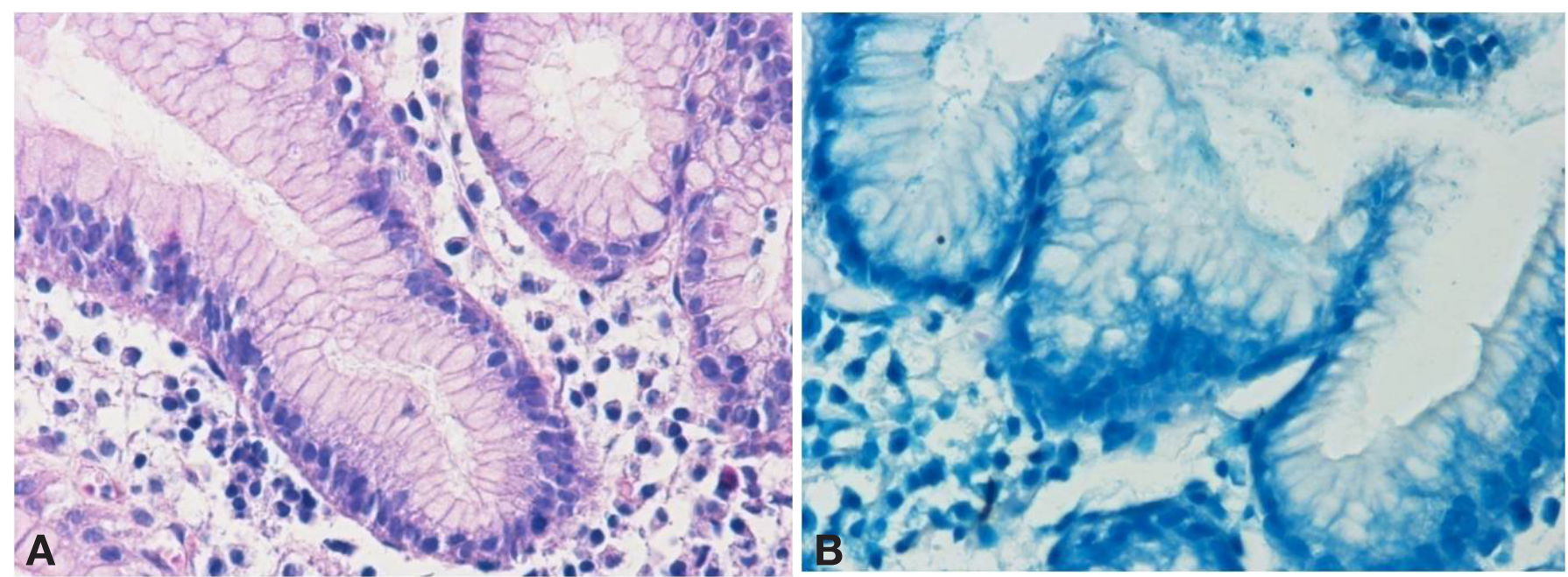

Fig. 1. Helicobacter pylori present in gastric foveolae in HE (A) and Giemsa stained slides (B). 
only in the corpus and in $90(6.61 \%)$ cases lesions were found in both areas. The statistical analysis points out a significant difference in reference to the frequency of the diagnosed predisposing and premalignant lesions in relation to the type of endoscopic biopsy (p-0.001), these lesions representing $41.90 \%$ of the total $\mathrm{N}=1890$ biopsies obtained from one region compared to $88.28 \%$ of total $\mathrm{N}=640$ obtained from two different areas of the gastric mucosa.

We found no statistically significant difference regarding gender distribution $(\mathrm{p}=0.4)$ of patients diagnosed with predisposing and premalignant lesions, gender ratio being $\mathrm{M}: \mathrm{F}=1: 1.05$. The mean age of patients with these lesions was 64.14 years, the most affected age group being 60-69 yo $(32.54 \%)$, with a statistically significant correlation between the two evaluated parameters $(\mathrm{p}=0.001)$, the diagnosis of these lesions being more frequent in age groups over 50 yo. The comparative analysis of the two study groups, under and over 50 yo, shows that only $7.31 \%$ of lesions were found in the first group, while the second group included $92.69 \%$ of cases.

We obtained a statistically significant difference between age groups, HP infection standing out for the under 50 yo

Table II. Helicobacter pylori present in gastric foveolae in HE (A) and Giemsa stained slides (B).

\begin{tabular}{ccccc}
\hline & $\mathrm{A}+$ & $\mathrm{A}-$ & $\mathrm{IM}+$ & $\mathrm{IM}-$ \\
\hline $\mathrm{HP}+$ & 24 & \multirow{2}{*}{$554(29.19 \%)$} & 208 & 948 \\
$\mathrm{p}-0.7$ & $(1.26 \%)$ & & $(5,27 \%)$ & $(25,18 \%)$ \\
\hline $\mathrm{HP}-$ & 92 & \multirow{2}{*}{$1228(64.70 \%)$} & 440 & 2200 \\
$\mathrm{p}-0.1$ & $(4.85 \%)$ & & $(11,17 \%)$ & $(58,38 \%)$ \\
\hline
\end{tabular}

Table III. HP infection and the correlation with the most frequent tupes of predisposing lesions found in biopsies taken from two different gastric regions.

\begin{tabular}{ccccc}
\hline & & $\mathrm{HP}-$ & $\mathrm{HP}+$ & $\mathrm{TOTAL}$ \\
\hline & & 298 & 196 & 494 \\
$\mathrm{~A}$ & - & $(46.6 \%)$ & $(26.6 \%)$ & $(77.2 \%)$ \\
\cline { 2 - 5 } $\mathrm{p}-0,7$ & & 93 & 57 & 146 \\
& + & $(14.0 \%)$ & $(8.8 \%)$ & $(22.8 \%)$ \\
\hline \multirow{2}{*}{$\mathrm{IM}$} & - & 548 & 334 & 882 \\
$\mathrm{p}-0,9$ & & $(41.84 \%)$ & $(26.86 \%)$ & $(68.7 \%)$ \\
\cline { 2 - 5 } & & 228 & 170 & 398 \\
& & $(18.78 \%)$ & $(12.52 \%)$ & $(31.3 \%)$ \\
\hline
\end{tabular}

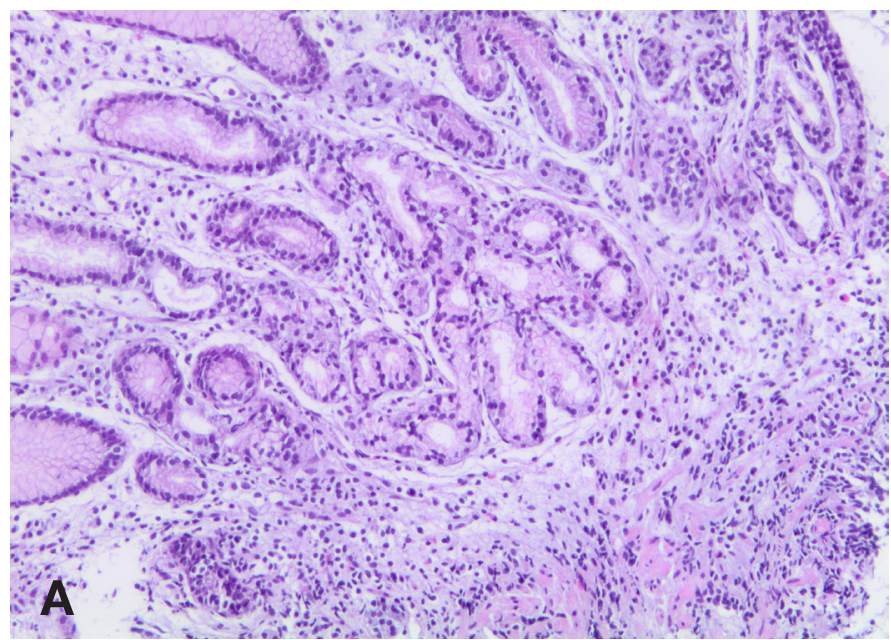

group ( $\mathrm{p}=0.001)$. Thus, HP infection was found in $23 \%$ of the total $\mathrm{N}=830$ relative to $7.31 \%$ of the total $\mathrm{N}=1357$ of cases with predisposing and premalignant lesions.

The results concerning the correlation between HP infection and the A and IM (Figure 2) found in biopsies taken from a single gastric area versus those taken from two different gastric areas are shown in the tables below (Table II) and (Table III).

PPM was found in $17(1.25 \%)$ cases, of which 4 (23.53\%) associated HP infection, with no statistically significant difference between the two parameters $(\mathrm{p}=0.47)$.

Most cases, 1334 (98.37\%), were included in the category negative for IEN, 17 (73.91\%) of cases being diagnosed with I-IEN, represented by hyperproliferative intestinal metaplasia, and $6(20.09 \%)$ cases being included in the LG-IEN category (Figure 3). HP infection was present in two cases of I-IEN and LG-IEN, respectively. Statistical analysis shows that in those cases diagnosed with IEN HP infection is absent thus proving a statistically significant negative correlation between the two $(p=0.002)$.

\section{Discussions}

Although all countries around the world are reporting a decrease in the incidence of intestinal type gastric cancer, it still remains an important public health problem, representing the second cause of death through cancer at global level [11-13] According to the gastric carcinogenesis pattern proposed in 1975 by Correa $\mathrm{P}$ et al. $[9,11,14]$ intestinal type gastric carcinoma is the end result of progressive, cascading alterations that occur at gastric mucosa level, with Helicobacter pylori having a pivotal role in their development.

Epidemiological studies show that chronic inflammation caused by this infection is the trigger factor of these successive events, adding to the environmental and genetic status of each individual and together being the determining factors of gastric cancer development $[5,6,13]$. In our country, where gastric cancer incidence is notably high $10.41 \%$ [15] similar to that of Portugal (13.06\%), Bulgaria $(10.3 \%)$, Croatia $(9.92 \%)$, there are no epidemiological

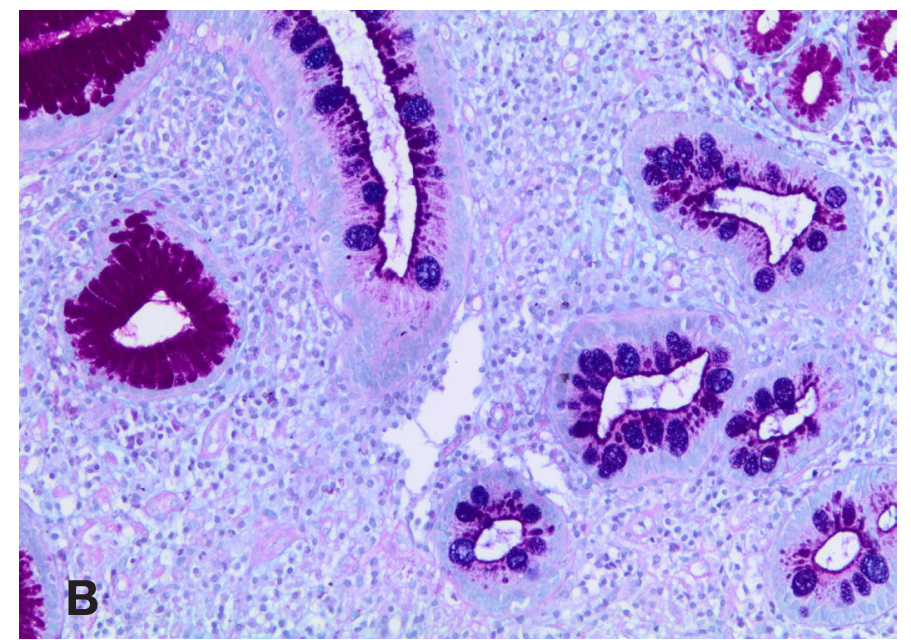

Fig. 2. Predisposing gastric lesions - glandular atrophy, HE 10x (A), intestinal metaplasia, PAS-AB, 20x (B) 


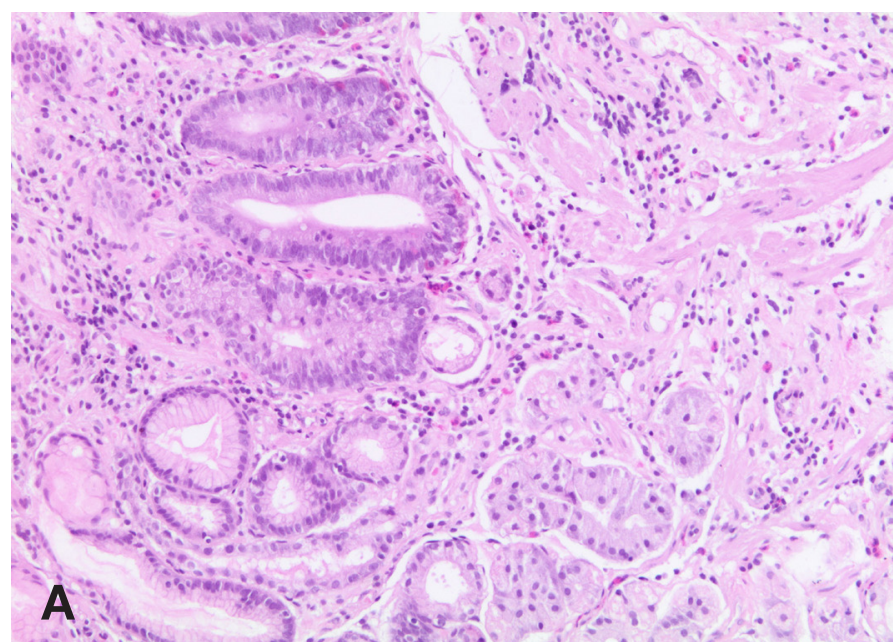

Fig. 3. Premalignant lesions - I-IEN, HE 10x (A), LG-IEN, HE 20x (B).

studies concerning the HP infection incidence [16]. Moreover, there is no data to support a correlation between this infection and the predisposing and premalignant lesions, and implicitly the gastric carcinoma, it induces.

Some studies carried out in Portugal, considered a country with a high incidence of gastric cancer, showed that Helicobacter pylori infection is close to $85 \%$ [17]. In comparison, our results prove that the percentage of HP positive cases is just $32.81 \%$ although according to data given by the International Agency for Research on Cancer (IARC) on the incidence of gastric cancer, Romania is included in the same category as Portugal [16]. The lower incidence of HP infection, in a country considered high risk for gastric cancer, supports the theory which states that, in the pathogenesis of gastric cancer, other factors are of high importance, especially those linked to nutrition and dieting. On the other hand, we must consider the fact that due to the non-specific symptoms, many patients are treated before diagnosis with proton pump inhibitors (PPI) or are prescribed antibiotics with effect on HP, determining its migration or disappearance, but maintaining inflammation $[9,18,19]$.

Determination of HP infection is also possible through other tests, such as the urease test, and, by this, some patients with a positive test are getting treated without going through a digestive endoscopy procedure during which biopsies are taken $[20,21]$. We can also state that there is no significant difference $(\mathrm{p}=0,35)$ between biopsies taken from a single location or from different areas of the stomach. This is due to the fact that HP initially affects the antrum but then it will also colonise the corpus.

The cases in which HP infection is positive only in the corpus stand out. In the situation in which the biopsy is taken only from the corporeal area, 14 cases in our study, and in which determining HP infection at antral level was not possible, the information brought by these cases is irrelevant. In the category of biopsies prelevated from different regions of the gastric mucosa, our study found 6 cases in which HP infection was only present in the corpus. In this type of cases, the extensive atrophic and/or

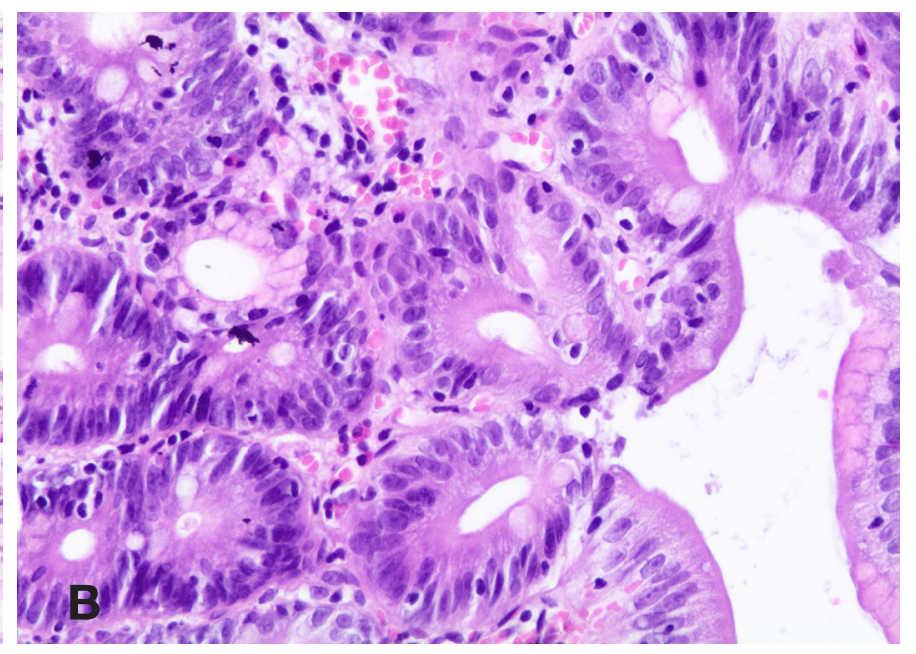

metaplastic alterations present only in the antrum, like in the cases with chronic PPI treatment, lead to a change in microclimate that translates intro the disappearance of the pathogen from the antrum and its migration towards the corporeal region. For these patients HP eradication is recommended without the need for periodical follow-up [22]. If, besides the HP infection found at corporeal level, there are predisposing lesions in both regions, these cases fall into the extensive lesion category, needing periodic follow-up involving upper gastrointestinal endoscopy and biopsies [23-25]. A final category of patients is consisted of those patients who have an HP infection and predisposing lesions exclusively in the corporeal area, representing those with chronic, autoimmune atrophic gastritis, caused by the infection [26-28]. In our study, we found no cases of predisposing lesions limited to the corporeal mucosa, suggestive aspect for an autoimmune gastritis and which, in most cases, is not linked to HP infection.

Our data shows that approximately half $(53.64 \%)$ of evaluated biopsies had predisposing or premalignant lesions. Notably, in $90(6.61 \%)$ cases that made it possible to evaluate both regions of the mucosa, we found lesions in both antral and corporeal areas. These cases may represent the category of patients with extensive, multifocal lesions for which guidelines recommend a 3 year apart followup for endoscopic evaluation with several biopsies being obtained from several gastric regions $[22,23,29,30]$. The significant difference between the frequency of predisposing and premalignant lesions as influenced by the type of endoscopic biopsy $(\mathrm{p}=0.001)$ can be explained by the fact that at least two biopsies from two different areas allows a better evaluation of the presence and extension of these lesions in the biopsies

The significant difference ( $\mathrm{p}-0.001)$ between the percentage of cases diagnosed with HP (23\%) and the percentage of diagnosed premalignant lesions $(7.31 \%)$ for the under 50 yo age group, shows that the infection occurs at a younger age but lesions develop in time and are manifest in older patients [31,32]. The fact that we found no correlation between HP frequency and the frequency of 
predisposing and premalignant lesions diagnosed on biopsies shows that in the gastric carcinogenesis other factors intervene, at least in our are, probably linked to the environment and food habits [2,5,6,33].

IEN lesions do not correlate with the presence of HP in biopsies, because LG-IEN represent late alterations that arise within a multifocal atrophic gastritis with metaplasia extended in both antrum and corpus. These extensive lesions lead to hypoacidity and the disappearance of HP. Concerning lesions I-IEN, most of the time, the cytonuclear changes are more pronounced because of HP infection and of the polimorphic inflammatory infiltrate. This is why these cases must be re-evaluated after the eradication treatment is finished.

\section{Conclusions}

The prevalence of HP infection in our study does not uphold our countrys' position in the ranking of countries with a high incidence of gastric cancer and proves that other regional, nutritional and life style factors are involved. This statement is also confirmed by the absence of a significant correlation between HP positive cases and the existance of predisposing and premalignant lesions in the studied casuistry.

With a difference in the under 50 yo age group between the number of HP infection cases and those with predisposing and premalignant lesions, we can state that there is a period of latency between the time of infection and the appearance of the lesions. This is also sustained by the fact that chronic inflammation is significantly associated with predisposing lesions.

The biopsies obtained from different regions of the gastric mucosa do not offer extra information regarding HP infection prevalence but prove their respective worth in the field of evaluating incidence and extension of predisposing and premalignant lesions.

\section{Acknowledgment}

This paper was published under the frame of $\mathrm{Eu}$ ropean Social Found, Human Resources Development Operational Programme 2007-2013, project no. POSDRU/159/1.5/S/136893

\section{References}

1. Kang JM, Shin DW, Kwon MY, et al. Stomach cancer screening and preventive behaviors in relatives of gastric cancer patients. World $\mathrm{J}$ Gastroenterol 2011;17(30):35183525.doi:10.3748/wjg.v17.i30.3518.

2. Karimi P, Islami F, Anandasabapathy S, Freedman ND, Kamangar F. Gastric cancer: descriptive epidemiology, risk factors, screening, and prevention. Cancer Epidemiol Biomarkers Prev. 2014;23(5):700-13. doi:10.1158/1055-9965.EPI-13-1057.

3. Lee YC, Chen TH, Chiu HM, et al. The benefit of mass eradication of Helicobacter pylori infection: a community-based study of gastric cancer prevention. Gut.2013;62(5):676-82.doi: 10.1136/gutjnl-2012-302240.

4. Pasechnikov V, Chukov S, Fedorov E, Kikuste I, Leja M. Gastric cancer: Prevention, screening and early diagnosis World $\mathrm{J}$ Gastroenterol. 2014;20(38):13842-13862. doi:10.3748/wjg.v20.i38.13842.

5. Jennifer MY, Sue J.G, Karen MK, Majid E. Effects of Helicobacter pylori infection and smoking on gastric cancer incidence in China: a population-level analysis of trends and projections Cancer Causes
Control. 2009;20(10): 2021-2029.doi:10.1007/s10552-009-9397-9.

6. Lee $\mathrm{YY}$, Derakhshan $\mathrm{MH}$. Environmental and lifestyle risk factors of gastric cancer. Arch Iran Med. 2013;16(6):358-65.doi: 013166/ AIM.0010.

7. Machado AM, Figueiredo C, Seruca R, Rasmussen LJ. Helicobacter pylori infection generates genetic instability in gastric cells. Biochim Biophys Acta. 2010;1806(1):58-65.doi: 10.1016/j.bbcan.2010.01.007.

8. Uemura N, Okamoto S, Yamamoto S, et al. Helicobacter pylori infection and the development of gastric cancer. N Engl J Med. 2001;345(11):7849.doi:10.1056/NEJMoa001999.

9. Watari J, Chen N, Amenta SP, et al. Helicobacter pylori associated chronic gastritis, clinical syndromes, precancerous lesions, and pathogenesis of gastric cancer development. World J Gastroenterol. 2014;20(18):5461-5473.doi:10.3748/wjg.v20.i18.5461.

10. Correa P. A human model of gastric carcinogenesis. Cancer Res 1988;48:3554-3560.

11. Correa P, Piazuelo MB, The gastric precancerous cascade. J Dig Dis 2012;13(1):2-9.doi: 10.1111/j.1751-2980.2011.00550.x.

12. Zheng L, Wang L, Ajani J, Xie K. Molecular basis of gastric cancer development and progression. Gastric Cancer 2004;7(2):61-77. doi10.1007/s10120-004-0277-4.

13. Brenner $\mathrm{H}$, Rothenbacher $\mathrm{D}$, Arndt $\mathrm{V}$. Epidemiology of stomach cancer. Methods Mol Biol 2009;472:467-477.doi:10.1007/978-1-60327-4920_23.

14. Correa P, Haenszel W, Cuello C, Tannenbaum S, Archer M. A model for gastric cancer epidemiology. Lancet, 1975;2:58-60.

15. Jernal A, Bray F, Center MM, et al. Globan cancer statistics. CA Cancer J Clin 2011;61:69-90.doi:10.3322/caac.20107.

16. http://globocan.iarc.fr/Pages/Map.aspx

17. Bastos J1, Peleteiro B, Barros R, et al. Sociodemographic determinants of prevalence and incidence of Helicobacter pylori infection in Portuguese adults. Helicobacter. 2013;18(6):413-22.doi:10.1111/hel.12061.

18. Saniee P, Shahreza S, Siavoshi F. Negative effect of proton-pump inhibitors (PPIs) on helicobacter pylori growth, morphology, and urease test and recovery after PPI removal - an in vitro study. Helicobacter. 2015;28.doi:10.1111/hel.12246.

19. Peleteiro B, Bastos A, Ferro A, Lunet N. Prevalence of Helicobacter pylori infection worldwide: a systematic review of studies with national coverage. Dig Dis Sci. 2014;59(8):1698-709.doi:10.1007/s10620-0143063-0.

20. Ogata SK, Kawakami E, Patrício FR, Pedroso MZ, Santos AM. Evaluation of invasive and non-invasive methods for the diagnosis of Helicobacter pylori infection in symptomatic children and adolescents. Sao Paulo Med J. 2001;119(2):67-71.doi.org/10.1590/S1516-31802001000200006.

21. Hahn M, Fennerty MB, Corless CL, et al. Noninvasive tests as a substitute for histology in the diagnosis of Helicobacter pylori infection. Gastrointest Endosc. 2000;52(1):20-6. doi:http://dx.doi.org/10.1067/ mge.2000.106686.

22. Dinis-Ribeiro M, Areia $M$, de Vries $A C$, Marcos-Pinto $R$, et al. Management of precancerous conditions and lesions in the stomach (MAPS): guideline from the European Society of Gastrointestinal Endoscopy (ESGE), European Helicobacter Study Group (EHSG), European Society of Pathology (ESP), and the Sociedade Portuguesa de Endoscopia Digestiva (SPED). Endoscopy 2012;44:74-94. doi:10.1055/s-0031-1291491.

23. Zullo A, Hassan C, Romiti A, et.al. Follow-up of intestinal metaplasia in the stomach: When, how and why. World J Gastrointest Oncol 2012;4(3):3036.doi:10.4251/wjgo.v4.i3.30.

24. Busuttil RA, Boussioutas A. Intestinal metaplasia: a premalignant lesion involved in gastric carcinogenesis. J Gastroenterol Hepatol 2009;24:193-201.doi:10.1111/j.1440-1746.2008.05774.x.

25. Kyrlagkitsis I, Karamanolis DG. Premalignant lesions and conditions for gastric adenocarcinoma: diagnosis, management and surveillance guidelines. Hepatogastroenterology. 2003;50(50):592-600.doi:10.5754/ hge121268.

26. Lea IV, Aino MO, Pentti IS, Hilpi IKR. Association of autoimmune type atrophic corpus gastritis with Helicobacter pylori infection. World J Gastroenterol. 2010;16(1):83-88.doi:10.3748/wjg.v16.i1.83.

27. Faller G1, Kirchner T. Immunological and morphogenic basis of gastric mucosa atrophy and metaplasia. Virchows Arch. 2005;446(1):1-9. doi10.1007/s00428-004-1157-3.

28. Cassaro M, Rugge M, Gutierrez O, et al. Topographic patterns of intestinal metpalasia and gastric cancer. Am J Gastroenterol 2000;95(6):1431-8doi:10.1038/ajg.2015.169.

29. Correa P, Piazuelo MB, Wilson KT. Pathology of gastric intestinal metaplasia: clinical implications. Am J Gastroenterol 2010;105(3):493-8.doi:10.1038/ajg.2009.728. 
30. Eriksson NK, Kärkkäinen PA, Färkkilä MA, Arkkila PE. Prevalence and distribution of gastric intestinal metaplasia and its subtypes.Dig Liver Dis 2008;40(5):35560.doi:10.1016/j.dld.2007.12.012.

31. Zhang C, Yamada N, Wu YL, et al. Helicobacter pylori infection glandular atrophy and intestinal metaplasia in superficial gastritis, gastric erosion, erosive gastritis, gastric ulcer and early gastric cancer. World $J$ Gastroenterol. 2005;14;11(6):791-6.doi:10.3748/wjg.v11.i6.791.
32. Pacifico L, Anania C, Osborn JF, Ferraro F, Chiesa C. Consequences of Helicobacter pylori infection in children. World J Gastroenterol. 2010;16(41):51815194.doi:10.3748/wjg.v16.i41.5181.

33. Wroblewski LE, Peek RM Jr, Wilson KT. Helicobacter pylori and gastric cancer: factors that modulate disease risk. Clin Microbiol Rev. 2010;23(4):713-39.doi:10.1128/CMR.0001110. 